\title{
BMJ open Dental status and incident falls among older Japanese: a prospective cohort study
}

\author{
Tatsuo Yamamoto, ${ }^{1}$ Katsunori Kondo, ${ }^{2}$ Jimpei Misawa, ${ }^{2}$ Hiroshi Hirai, ${ }^{3}$ \\ Miyo Nakade, ${ }^{4}$ Jun Aida, ${ }^{5}$ Naoki Kondo, ${ }^{6}$ Ichiro Kawachi, ${ }^{7}$ Yukio Hirata ${ }^{1}$
}

To cite: Yamamoto $\mathrm{T}$, Kondo $\mathrm{K}$, Misawa J, et al. Dental status and incident falls among older Japanese: a prospective cohort study. BMJ Open 2012;2:e001262. doi:10.1136/

bmjopen-2012-001262

- Prepublication history for this paper is available online. To view this file please visit the journal online (http://dx.doi.org/10.1136/ bmjopen-2012-001262)

Received 4 April 2012 Accepted 5 July 2012

This final article is available for use under the terms of the Creative Commons Attribution Non-Commercial 2.0 Licence; see http://bmjopen.bmj.com

For numbered affiliations see end of article.

Correspondence to Dr Yukio Hirata; hiratay@kdcnet.ac.jp

\section{ABSTRACT}

Objective: To examine if self-reported number of teeth, denture use and chewing ability are associated with incident falls.

Design: Longitudinal cohort study (the Aichi Gerontological Evaluation Study).

Setting: 5 Japanese municipalities.

Participants: 1763 community-dwelling individuals aged 65 years and older without experience of falls within the previous year at baseline.

Main outcome measures: Self-reported history of multiple falls during the past year at the follow-up survey about 3 years later. Baseline data on the number of teeth present and/or denture use and chewing ability were collected using self-administered questionnaires. Logistic regression analyses controlled for sex, age, functional disability during follow-up period, depression, self-rated health and educational attainment.

Results: 86 (4.9\%) subjects reported falls at the follow-up survey. Logistic regression models fully adjusted for all covariates showed that subjects having 19 or fewer teeth but not using dentures had a significantly increased risk for incident falls $(O R$ $2.50,95 \% \mathrm{Cl} 1.21$ to $5.17, \mathrm{p}=0.013$ ) compared with those having 20 or more teeth. Among subjects with 19 or fewer teeth, their risk of falls was not significantly elevated so long as they wore dentures (OR $1.36,95 \% \mathrm{Cl} 0.76$ to $2.45, \mathrm{p}=0.299$ ). No significant association was observed between chewing ability and incident falls in the fully adjusted model.

Conclusions: Having 19 or fewer teeth but not using dentures was associated with higher risk for the incident falls in older Japanese even after adjustment for multiple covariates. Dental care to prevent tooth loss and denture treatment for older people might prevent falls, although the authors cannot exclude the possibility that the association is due to residual confounding.

\section{INTRODUCTION}

Falls occur frequently in older people and adversely affect their quality of life. More than one-third of persons aged 60 years and older fall each year in Japan, ${ }^{1}$ England $^{2}$ and

\section{ARTICLE SUMMARY}

Article focus

- An association has been reported between dental occlusion and physical function including lower extremity dynamic strength and balance.

- Whether number of teeth, denture use and chewing ability predict subsequent incidence of falls is unknown.

- The aim of this study was to examine if selfreported number of teeth, denture use and chewing ability are associated with incident falls.

Key messages

- Having 19 or fewer teeth but not using dentures was a strong independent predictor of incident falls in a community-dwelling older population.

- In addition to preventing tooth loss, denture treatment for older people might prevent falls.

Strengths and limitations of this study

- Strengths of this study include large sample size, population-based sampling and control for many potential confounding factors.

- The following limitations should be considered: the information on dental status and falls was self-reported and misclassification of cases is possible. Moreover, we cannot exclude the possibility that the association is due to residual confounding.

the USA, ${ }^{3}$ and in half of cases, falls are recurrent. ${ }^{3}$ The consequences of falls are severe: $6 \%$ of falls lead to a fracture and $24 \%$ lead to other serious injuries. ${ }^{2}$ Thus, falls impose a burden on the sustainability of health and long-term care in Japan and many other countries, where population is rapidly ageing. ${ }^{4}$ In Japan, the annual health and long-term care costs attributable to falls are about 730 thousand million Japanese yen in 2002 , which amounts to roughly $5 \%$ of the entire health and long-term care costs. ${ }^{5}$

A number of studies ${ }^{6}$ including those using Japanese data ${ }^{7}$ have identified risk factors for falls, including female sex, older age, having a history of falls, arthritis, cerebrovascular 
disease, depression and the impairment of muscle strength and/or balance. Although exercise programmes including balance training are effective, ${ }^{8}$ multifactorial fall prevention programmes to address these risk factors have not been successful in reducing falls. ${ }^{9}$ A recent systematic review concluded that there is limited evidence to suggest that multifactorial fall prevention programmes in primary care, community or emergency care settings are effective in reducing the number of falls. ${ }^{10}$ Therefore, identification of additional modifiable risk factors may be helpful to establish more effective programmes for fall prevention.

Unhealthy dental status is a candidate risk factor for falls. ${ }^{11}{ }^{12}$ A longitudinal study showed that partial or complete loss of dental occlusion was associated with a decline in lower extremity dynamic strength and balance function. ${ }^{13}$ One 1-year prospective longitudinal study using 146 demented older people reported the association between dental occlusion and subsequent physical health. ${ }^{14}$ Researchers have argued that these potential links between dental health and physical health may be because jaw position affects body posture. ${ }^{11}$ Proprioceptive receptors of the masticatory muscular system and dentoalveolar ligaments provide sensory afferent input, ${ }^{15}$ and hence poor dental occlusion may decrease that proprioception, thereby interfering with the stability of head posture (and increasing the risk of falling). However, whether or not dental occlusion and chewing ability actually predict the subsequent incidence of falls is largely unknown.

Therefore, this prospective study aimed to determine the association between dental health in terms of the number of teeth present, denture use and chewing ability and the incidence of falls in a large cohort of older Japanese people.

\section{METHODS}

\section{Study population}

Our analyses were based on data from the Aichi Gerontological Evaluation Study (AGES) Project, an ongoing Japanese prospective cohort study. ${ }^{16}{ }^{17}$ The detailed protocol of the AGES and baseline characteristics of the study participants have been published elsewhere. ${ }^{16} 18$ In brief, the AGES aims to investigate the factors related to the loss of healthy years, such as functional decline, cognitive impairment or death among non-institutionalised older people. The sample was restricted to those who did not already have a physical or cognitive disability at baseline, defined by not receiving public long-term care insurance benefits and self-reported dependence in walking, toileting and bathing.

The sampling frame for the AGES cohort was selected as follows. In 2003, the residential registers of five municipalities in Aichi prefecture were obtained with the cooperation of city officials. From these comprehensive registers, we selected a random sample of one in three citizens aged 65 years or older in four towns (1281,
1537,1766 and 1873) and a random sample of 1666 in a city. They $(\mathrm{N}=8123)$ were then mailed the baseline questionnaire, inviting them to participate in the AGES cohort study. Responses were obtained from 3998 subjects $(49.2 \%)$, and 3981 subjects were identified using ID. We mailed a follow-up survey between March 2006 and March 2007 to the 3471 subjects after excluding 510 subjects who died or started receiving insurance benefits due to certified disability $(\mathrm{N}=472)$ or could not be traced $(\mathrm{N}=38)$. Two thousand six hundred and forty subjects responded to the follow-up survey $(76.1 \%)$ and formed the analytic sample for our study. After excluding 166 and 545 subjects who experienced multiple and single falls, respectively, as well as 106 subjects without information of falls at baseline, we were left with 1823 subjects who did not report experiencing falls at baseline. After excluding 56 subjects without information on falls at follow-up and four subjects without information on age, a total of 1763 subjects formed the final analytic population of this study. The AGES protocol was reviewed and approved by the Ethics Committee on Research of Human Subjects at Nihon Fukushi University.

\section{Outcome variables}

History of falls was ascertained by asking, "Have you had any falls over the past year?" with possible answers of "multiple times," "once" or "none." Multiple falls was used as an outcome and the last two categories were combined because previous studies have found that single fallers are more similar to non-fallers than to recurrent fallers on a range of medical, physical and psychological risk factors. ${ }^{3} 1920$

\section{Dental health variables}

Dental status and chewing ability were assessed using a self-administered questionnaire. Respondents were asked to classify their dental status as having 20 or more teeth, having 19 or fewer teeth with dentures, having 19 or fewer teeth without dentures, having few teeth with dentures or having few teeth without dentures. Data from having 19 or fewer teeth with dentures and those from having few teeth with dentures were combined. Data from having 19 or fewer teeth without dentures and those from having few teeth without dentures were also combined.

Chewing ability was ascertained by asking, "How is your ability to chew?" with possible answers being "I can chew anything I want," "I can chew most foods with some exceptions," "I can eat limited foods as I cannot chew very well," "I can hardly chew anything" and "I have liquid foods as I cannot chew at all." Data from the last three categories were combined due to the small number of respondents.

\section{Covariates}

Studies suggest that falls are associated with sex,${ }^{721}$ age,${ }^{27}$ stroke, ${ }^{7}$ severe foot problems, ${ }^{2}$ impaired vision and impaired hearing, ${ }^{2}$ activities of daily living (ADL), ${ }^{3} 621$ 
body mass index (BMI) ${ }^{21}$ use of sedatives, ${ }^{2}$ depression, ${ }^{2}$ self-rated health, ${ }^{21}$ exercise, ${ }^{2}$ mobility ${ }^{2}$ and socioeconomic status. ${ }^{22}$ Therefore, associations of incident fall with sex, age, present illness related to falls, ADL, BMI, use of sedatives, depression, self-rated health, exercise (how much they walked in minutes per day), frequency of outings, educational attainment and equivalised household income were analysed. Selfreported current medical treatment of stroke, osteoporosis, joint disease/neuralgia, injury/fracture, impaired vision and/or impaired hearing was used as a variable for present illness related to falls. To evaluate functional status, the survey asked whether the respondents had difficulty or needed someone's assistance in performing any of the following ADL: basing, walking and using the toilet. ${ }^{23}$ Subjects without difficulty for all three ADL items were categorised into ADL without limitation and those with at least one ADL item with difficulty into ADL with limitation. BMI was categorised into three groups $(<18.5,18.5-24.9$ and 25.0 or more). ${ }^{24}$ Depression was assessed with the short version of the Geriatric Depression Scale-15 developed for self-administration in the community using a simple yes/no format ${ }^{25}$ and was categorised into three groups: 0-4 (no), 5-9 (mild) and 10-15 (moderate to severe). To adjust household income for household size, equivalised income was calculated by dividing the household income by the square root of the number of household members and grouped into one of the seven categories. In addition to these covariates, data on functional disability during follow-up period were collected from the public long-term care insurance database maintained by each participating municipality and used as a covariate. Incidence of functional disability was determined based on when a person newly qualified for the insurance benefit, new registrations to the public long-term care insurance database. ${ }^{26}$ The distribution of each covariate at baseline for the overall AGES 2003 cohort $(n=32891)$ has been reported elsewhere. ${ }^{16}$

\section{Statistical analysis}

Categorical variables that included missing data were recorded by reassigning missing values to separate "missing" categories in order to maximise the number of subjects included in the statistical analysis and thereby maximise statistical power. Logistic regression models were used to calculate the ORs and 95\% CIs for the incident falls at the follow-up. First, univariate ORs were calculated for each dental health variable and each covariate. Variables that were marginally significant $(p<0.10)$ in the univariate analyses were selected as covariates for subsequent multivariate analysis. Then, logistic regression analysis was performed for each dental variable after including sex, age, functional disability during follow-up period, depression, self-rated health and educational attainment as covariates. All statistical analyses were conducted using IBM SPSS Statistics V.19 (IBM Co.).

\section{RESULTS}

A total of $86(4.9 \%)$ of the 1763 respondents reported the incidence of multiple falls at the follow-up survey. Table 1 shows the rates of fallers and non-adjusted ORs for reporting multiple falls at the follow-up survey according to dental health variables and covariates. Univariate models showed that poor dental status, chewing ability, male sex, older age, lower functional disability during follow-up period, depression, poor selfrated health and low educational attainment were each associated with incident falls.

In the fully adjusted model, subjects with 19 or fewer teeth without dentures had a 2.50 (95\% CI 1.21 to 5.17)fold increased risk for incident falls compared with those having 20 or more teeth(Table 2). No significant association was observed between incident falls and chewing ability after adding all covariates in the logistic regression model. When the two dental health variables were entered simultaneously into the same model with full adjustment for all covariates, there was no change in the ORs for any of the dental status variables.

\section{DISCUSSION}

Results of the present study showed that subjects with 19 or fewer teeth without dentures had a significantly higher risk for incident falls than those with 20 or more teeth, even after adjusting for multiple potential confounding factors including demographics, physical and mental health status, and socioeconomic status. These findings are consistent with those of a 1-year longitudinal study using 146 older patients with severe dementia, showing that patients with functionally inadequate dental status had significantly more frequent falls than those with functionally adequate occlusion composed of natural teeth, dentures or both. ${ }^{14}$ Interestingly, among subjects with 19 or fewer teeth, their risk of falls was not significantly elevated so long as they wore dentures. These results suggest that the poor dental occlusion due to not using dentures after losing teeth is a strong risk factor for falls among subjects with 19 or fewer teeth.

There are several possible pathways between not using dentures after losing teeth and incident falls. One possibility is that the loss of occlusion due to not using dentures may result in a decrease in functional balance and these functional declines lead to falls. A crosssectional study suggests that dental occlusal condition is associated with balance function. ${ }^{12}$ An 8 -year longitudinal study showed that partial or complete loss of occlusion was associated with a decrease in balance function. ${ }^{13}$ Because balance deficit is a well-known risk factor for falls, ${ }^{6}$ it is plausible that poor balance may explain the increased risk of falls among subjects with poor dental occlusion.

A clinical study showed that dental occlusion affects postural and gaze stabilisation because proprioceptive receptors of the masticatory muscular system and dentoalveolar ligaments provide sensory afferent input, 
Table 1 Univariate associations of dental health variables and covariates with incident falls

\begin{tabular}{|c|c|c|c|c|}
\hline & $\begin{array}{l}\text { Total } \\
\mathbf{n}\end{array}$ & $\begin{array}{l}\text { Fallers } \\
\text { n (\%) }\end{array}$ & OR $(95 \% \mathrm{Cl})$ & p Value \\
\hline \multicolumn{5}{|l|}{ Dental status } \\
\hline$\geq 20$ teeth & 586 & $17(2.9)$ & 1.00 & \\
\hline$\leq 19$ teeth with dentures & 958 & $49(5.1)$ & $1.80(1.03$ to 3.16$)$ & 0.039 \\
\hline$\leq 19$ teeth without dentures & 198 & $17(8.6)$ & 3.14 (1.57 to 6.28$)$ & 0.001 \\
\hline Missing & 21 & $3(14.3)$ & 5.58 (1.50 to 20.76$)$ & 0.010 \\
\hline \multicolumn{5}{|l|}{ Chewing ability } \\
\hline Can chew anything & 719 & $30(4.2)$ & 1.00 & \\
\hline Can chew most foods & 935 & $47(5.0)$ & $1.22(0.76$ to 1.94$)$ & 0.414 \\
\hline Cannot chew very well & 97 & $9(9.3)$ & 2.35 (1.08 to 5.11$)$ & 0.031 \\
\hline Missing & 12 & $0(0.0)$ & $0.00(0.00)$ & 0.999 \\
\hline \multicolumn{5}{|l|}{ Sex } \\
\hline Female & 853 & $32(3.8)$ & 1.00 & \\
\hline Male & 910 & $54(5.9)$ & 1.62 (1.03 to 2.53$)$ & 0.035 \\
\hline \multicolumn{5}{|l|}{ Age (years) } \\
\hline $65-69$ & 707 & $23(3.3)$ & 1.00 & \\
\hline $70-74$ & 569 & $27(4.7)$ & $1.48(0.84$ to 2.61$)$ & 0.175 \\
\hline $75-79$ & 325 & $18(5.5)$ & 1.74 (0.93 to 3.28$)$ & 0.084 \\
\hline $80-84$ & 120 & $12(10.0)$ & 3.30 (1.60 to 6.84$)$ & 0.001 \\
\hline$\geq 85$ & 42 & $6(14.3)$ & $4.96(1.90$ to 12.93$)$ & 0.001 \\
\hline \multicolumn{5}{|l|}{ Present illness related to falls* } \\
\hline No & 1224 & $58(4.7)$ & 1.00 & \\
\hline Yes & 539 & $28(5.2)$ & $1.10(0.69$ to 1.75$)$ & 0.682 \\
\hline \multicolumn{5}{|l|}{ Activities of daily living } \\
\hline Without limitation & 1669 & $81(4.9)$ & 1.00 & \\
\hline With limitation or missing & 94 & $5(5.3)$ & $1.10(0.44$ to 2.79$)$ & 0.838 \\
\hline \multicolumn{5}{|c|}{ Functional disability during follow-up period } \\
\hline No & 1734 & $81(4.7)$ & 1.00 & \\
\hline Yes & 29 & $5(17.2)$ & 4.25 (1.58 to 11.43$)$ & 0.004 \\
\hline \multicolumn{5}{|l|}{ Body mass index } \\
\hline$<18.5$ & 113 & $8(7.1)$ & 1.68 (0.78 to 3.62$)$ & 0.189 \\
\hline $18.5-24.9$ & 1196 & $52(4.3)$ & 1.00 & \\
\hline$\geq 25.0$ & 380 & $21(5.5)$ & 1.29 (0.77 to 2.17$)$ & 0.342 \\
\hline Missing & 74 & $5(6.8)$ & 1.59 (0.62 to 4.12$)$ & 0.336 \\
\hline \multicolumn{5}{|l|}{ Use of sedatives } \\
\hline No & 1602 & $80(5.0)$ & 1.00 & \\
\hline Yes & 161 & $6(3.7)$ & $0.74(0.32$ to 1.72$)$ & 0.478 \\
\hline \multicolumn{5}{|l|}{ Depression } \\
\hline No & 1143 & $39(3.4)$ & 1.00 & \\
\hline Mild & 311 & $21(6.8)$ & 2.05 (1.19 to 3.54$)$ & 0.010 \\
\hline Moderate to severe & 77 & $8(10.4)$ & 3.28 (1.48 to 7.29$)$ & 0.004 \\
\hline Missing & 232 & $18(7.8)$ & 2.38 (1.34 to 4.24$)$ & 0.003 \\
\hline \multicolumn{5}{|l|}{ Self-rated health } \\
\hline Excellent & 162 & $6(3.7)$ & 1.00 & \\
\hline Good & 1192 & $50(4.2)$ & $1.14(0.48$ to 2.70$)$ & 0.769 \\
\hline Fair & 321 & $21(6.5)$ & $1.82(0.72$ to 4.60$)$ & 0.206 \\
\hline Poor & 59 & $9(15.3)$ & 4.68 (1.59 to 13.80$)$ & 0.005 \\
\hline Missing & 29 & $0(0.0)$ & $0.00(0.00)$ & 0.998 \\
\hline \multicolumn{5}{|l|}{ Exercise (walk in minute/day) } \\
\hline$\geq 60$ & 430 & $20(4.7)$ & 1.00 & \\
\hline $30-59$ & 569 & $16(2.8)$ & $0.59(0.30$ to 1.16$)$ & 0.126 \\
\hline$<30$ & 564 & $38(6.7)$ & $1.48(0.85$ to 2.58$)$ & 0.167 \\
\hline Missing & 200 & $12(6.0)$ & 1.31 (0.63 to 2.73$)$ & 0.474 \\
\hline \multicolumn{5}{|l|}{ Frequency of outings } \\
\hline Almost everyday & 843 & $37(4.4)$ & 1.00 & \\
\hline $2-3$ times a week & 532 & $27(5.1)$ & $1.17(0.70$ to 1.94$)$ & 0.557 \\
\hline Once a week or less & 335 & $20(6.0)$ & 1.38 (0.79 to 2.42$)$ & 0.256 \\
\hline Missing & 53 & $2(3.8)$ & $0.85(0.20$ to 3.65$)$ & 0.831 \\
\hline
\end{tabular}




\begin{tabular}{|c|c|c|c|c|}
\hline & $\begin{array}{l}\text { Total } \\
\mathbf{n}\end{array}$ & $\begin{array}{l}\text { Fallers } \\
\text { n (\%) }\end{array}$ & OR $(95 \% \mathrm{Cl})$ & p Value \\
\hline \multicolumn{5}{|c|}{ Educational attainment (years) } \\
\hline$\geq 13$ & 184 & $4(2.2)$ & 1.00 & \\
\hline $10-12$ & 506 & $23(4.5)$ & $2.14(0.73$ to 6.28$)$ & 0.165 \\
\hline $6-9$ & 953 & $49(5.1)$ & $2.44(0.87$ to 6.84$)$ & 0.090 \\
\hline$<6$ & 53 & $4(7.5)$ & 3.67 (0.89 to 15.22$)$ & 0.073 \\
\hline Missing & 67 & $6(9.0)$ & $4.43(1.21$ to 16.21$)$ & 0.025 \\
\hline \multicolumn{5}{|c|}{ Equivalised household income (yen) } \\
\hline$<500000$ & 53 & $0(0.0)$ & $0.00(0.00)$ & 0.997 \\
\hline $500000-999999$ & 108 & $7(6.5)$ & 1.00 & \\
\hline $1000000-1499999$ & 139 & $7(5.0)$ & 0.77 (0.26 to 2.25$)$ & 0.627 \\
\hline $1500000-1999999$ & 262 & $15(5.7)$ & $0.88(0.35$ to 2.21$)$ & 0.780 \\
\hline $2000000-2999999$ & 429 & $16(3.7)$ & $0.56(0.22$ to 1.39$)$ & 0.213 \\
\hline $3000000-3999999$ & 263 & $15(5.7)$ & 0.87 (0.35 to 2.20$)$ & 0.773 \\
\hline$\geq 4000000$ & 164 & 5 (3.0) & $0.45(0.14$ to 1.47$)$ & 0.187 \\
\hline Missing & 345 & $21(6.1)$ & $0.94(0.39$ to 2.26$)$ & 0.882 \\
\hline
\end{tabular}

and hence poor dental occlusion may decrease that proprioception and interfere with the stability of head posture. ${ }^{15}$ Another clinical study showed that denture use improves postural swaying. ${ }^{27}$ Because using dentures reduced the OR for incident falls in the present study, proprioceptive receptors of the masticatory muscular system might be more strongly associated with balance function and falls than those of dentoalveolar ligaments.

Some subjects with 20 or more teeth may have had dentures in the present study; however, the information was not obtained. Subjects with 20 or more teeth without dentures may be more appropriate than those with 20 or more teeth with/without dentures as a reference, and lack of the information might underestimate the association between dental status and incident falls. However, studies show that people having at least 20 teeth usually can eat anything even they do not ware dentures. $^{28} 29$ Therefore, the lack of the information of with/without dentures in subjects having 20 or more teeth may be negligible.

We excluded individuals with history of falls because we wanted to examine prospectively the risk of incident falls. In addition, there is a theoretical possibility that history of falls might confound the association between number of teeth and risk of future falls, that is, history of falls in the past can be a prior common cause of (1) number of teeth (because some people may break teeth when they fall) and (2) past falls predict future falls. For these reasons, we felt that it was justified to exclude those with fall history at baseline.

In the present study, self-reported chewing ability was not associated with the incident falls. This result disagreed with those from a cross-sectional study showing a significant association between chewing ability judged from number of foods chewable and one-leg standing time. ${ }^{30}$ Because self-reported mastication can be modified by cooking (eg, cooking soft meal helps chewing ability) and is more subjective than selfreported number of teeth present and denture use, this might dilute the association between self-reported chewing ability and the incident falls. A study using 5643 subjects aged $40-89$ years showed that number of functional teeth which differentiate subjects with and without subjective dysphagia, defined as suffering any kind of subjective impairment to eating function, including biting difficulty, declined with age. ${ }^{31}$ Additional studies using objective measures for chewing ability are required to clarify the relationship between chewing ability and the incident falls.

Using dentures does not always recover chewing ability. For example, a cross-sectional study showed that biting forces among removable partial and complete denture wearers were $35 \%$ and $11 \%$, respectively, when expressed as a percentage of the subjects with natural dentition. $^{32}$ These results may explain the different associations of dental status and chewing ability with incident falls in the present study. When both dental status and chewing ability were simultaneously entered in the fully adjusted logistic regression model in the present study, only dental status was still significantly associated with incident falls.

Men were at increased risk of falls in the present study, which disagrees with a meta-analysis ${ }^{7}$ based on people aged 60 years or older showing that female sex was a risk factor. On the other hand, our study result is also similar to another large study of 12684 individuals aged 85 years or older. ${ }^{21}$ We feel that the association between sex and risk of falls might vary according to the study population. Indeed, current clinical guidelines for the prevention of falls do not include sex as a risk factor, ${ }^{63}$ and thus it may not be a settled question.

\section{Strengths and limitations}

We note some strength of the present study including large sample size, population-based sampling and 
Table 2 Multivariate adjusted OR and $95 \% \mathrm{Cl}$ for the association of dental status and chewing ability with incident falls

\begin{tabular}{|c|c|c|c|c|}
\hline & OR (95\% Cl) & p Value & OR $(95 \% \mathrm{Cl})$ & p Value \\
\hline \multicolumn{5}{|l|}{ Dental status } \\
\hline$\geq 20$ teeth & 1.00 & & & \\
\hline$\leq 19$ teeth with dentures & $1.36(0.76$ to 2.45$)$ & 0.299 & & \\
\hline$\leq 19$ teeth without dentures & $2.50(1.21$ to 5.17$)$ & 0.013 & & \\
\hline Missing & 5.75 (1.23 to 26.78$)$ & 0.026 & & \\
\hline \multicolumn{5}{|l|}{ Chewing ability } \\
\hline Can chew anything & & & 1.00 & \\
\hline Can chew most foods & & & 0.97 (0.59 to 1.59$)$ & 0.910 \\
\hline Cannot chew very well & & & 1.47 (0.64 to 3.37$)$ & 0.361 \\
\hline Missing & & & $0.00(0.00)$ & 0.999 \\
\hline \multicolumn{5}{|l|}{ Sex } \\
\hline Female & 1.00 & & 1.00 & \\
\hline Male & 1.86 (1.16 to 2.96$)$ & 0.010 & 1.86 (1.16 to 2.96$)$ & 0.009 \\
\hline \multicolumn{5}{|l|}{ Age (years) } \\
\hline $65-69$ & 1.00 & & 1.00 & \\
\hline $70-74$ & $1.31(0.73$ to 2.34$)$ & 0.366 & 1.36 (0.76 to 2.42$)$ & 0.302 \\
\hline $75-79$ & $1.42(0.74$ to 2.74$)$ & 0.290 & 1.57 (0.82 to 3.02$)$ & 0.178 \\
\hline $80-84$ & 2.51 (1.17 to 5.39$)$ & 0.018 & 2.84 (1.34 to 6.04$)$ & 0.007 \\
\hline$\geq 85$ & 3.78 (1.27 to 11.19$)$ & 0.017 & 4.63 (1.59 to 13.49$)$ & 0.005 \\
\hline \multicolumn{5}{|c|}{ Functional disability during follow-up period } \\
\hline No & 1.00 & & 1.00 & \\
\hline Yes & $2.30(0.75$ to 7.06$)$ & 0.144 & $2.11(0.70$ to 6.37$)$ & 0.184 \\
\hline \multicolumn{5}{|l|}{ Depression } \\
\hline No & 1.00 & & 1.00 & \\
\hline Mild & $1.82(1.01$ to 3.26$)$ & 0.046 & $1.82(1.01$ to 3.26$)$ & 0.045 \\
\hline Moderate to severe & $2.47(1.02$ to 5.97$)$ & 0.045 & 2.49 (1.03 to 6.02$)$ & 0.042 \\
\hline Missing & 2.14 (1.09 to 4.17$)$ & 0.026 & 2.07 (1.06 to 4.04$)$ & 0.032 \\
\hline \multicolumn{5}{|l|}{ Self-rated health } \\
\hline Excellent & 1.00 & & 1.00 & \\
\hline Good & $1.14(0.47$ to 2.77$)$ & 0.767 & 1.07 (0.44 to 2.59$)$ & 0.877 \\
\hline Fair & $1.43(0.54$ to 3.78$)$ & 0.474 & $1.33(0.50$ to 3.54$)$ & 0.568 \\
\hline Poor & 2.71 (0.83 to 8.77$)$ & 0.097 & 2.60 (0.81 to 8.34$)$ & 0.109 \\
\hline Missing & $0.00(0.00)$ & 0.998 & $0.00(0.00)$ & 0.998 \\
\hline \multicolumn{5}{|l|}{ Educational attainment (years) } \\
\hline$\geq 13$ & 1.00 & & 1.00 & \\
\hline $10-12$ & $2.49(0.83$ to 7.45$)$ & 0.102 & 2.59 (0.87 to 7.72$)$ & 0.089 \\
\hline $6-9$ & 2.21 (0.77 to 6.33$)$ & 0.140 & 2.50 (0.87 to 7.12$)$ & 0.087 \\
\hline$<6$ & 1.77 (0.37 to 8.56$)$ & 0.476 & $2.24(0.49$ to 10.27$)$ & 0.299 \\
\hline Missing & $2.78(0.68$ to 11.38$)$ & 0.156 & $3.17(0.77$ to 13.01$)$ & 0.110 \\
\hline
\end{tabular}

control for many potential confounding factors. However, the present study has a number of limitations. First, measurement of dental status was based on selfreport, not based on clinical examination. However, the validity and reliability of self-reported number of teeth has been established by multiple studies and widely used in epidemiological surveys. ${ }^{34}$ For example, validation studies in the USA and Japan have reported a high agreement between self-reported and examined number of teeth (Pearson's correlation coefficient: $r=0.97$ and 0.93 , respectively) in 50 community-dwelling individuals aged 70 years or older and 2496 subjects with a mean age of 59. ${ }^{35} 36$ Second, self-report of falls may not be perfectly accurate. ${ }^{37}$ However, the associations with demographic factors and other covariates are in the generally expected direction, suggesting that there may be sufficient value in this outcome.

\section{CONCLUSIONS}

The primary implication of this study is the importance of maintaining the dental occlusion, especially with natural teeth, in order to prevent falls among older adults. The loss of teeth might be an independent risk factor for incident falls, but it could be prevented by using dentures. Promoting dental care including proper use of denture might be an additional option for the prevention of falls in addition to current interventions targeting conventional risk factors, which warrants further interventional studies testing the effects of dental care and denture use on the prevention of falls.

Author affiliations

${ }^{1}$ Division of Sociological Approach in Dentistry, Department of Dental Sociology, Kanagawa Dental College, Yokosuka-shi, Kanagawa, Japan ${ }^{2}$ Center for Well-being and Society, Nihon Fukushi University, Nagoya-shi, Aichi, Japan 
${ }^{3}$ Department of Civil and Environmental Engineering, Faculty of Engineering, Iwate University, Morioka-shi, Iwate, Japan

${ }^{4}$ Department of Nutrition, Faculty of Health and Nutrition, Tokaigakuen University, Nagoya-shi, Aichi, Japan

${ }^{5}$ Department of International and Community Oral Health, Tohoku University Graduate School of Dentistry, Sendai-shi, Miyagi, Japan

${ }^{6}$ Department of Health Sciences, Interdisciplinary Graduate School of Medicine and Engineering, University of Yamanashi, Chuo-shi, Yamanashi, Japan ${ }^{7}$ Department of Society, Human Development and Health, Harvard School of Public Health, Boston, Massachusetts, USA

Contributors TY and YH had the idea for the study, participated in its design, performed the statistical analysis and drafted the manuscript, and $\mathrm{YH}$ is the guarantor. KK is coordinator of Aichi Gerontological Evaluation Study Project, helped develop the idea of the study, participated in acquiring the data and with design and edited the manuscript. JM participated in design of study and edited the manuscript. $\mathrm{HH}$ and MN participated in acquiring the data and with design and critically revised the manuscript. JA, NK and IK participated in the design of the study, deciding on statistical methods used, helped in interpreting the results and revising the manuscript.

Funding This study was supported in part by a grant of the Strategic Research Foundation Grant-aided Project for Private Universities from Ministry of Education, Culture, Sport, Science and Technology, Japan (MEXT), 2009-2013, and Health Labour Sciences Research Grant, Comprehensive Research on Aging and Health (H22-Choju-Shitei-008) from the Japanese Ministry of Health, Labour and Welfare.

Competing interests None.

Ethical approval The Aichi Gerontological Evaluation Study protocol including the present study was reviewed and approved by the Ethics Committee on Research of Human Subjects at Nihon Fukushi University.

Provenance and peer review Not commissioned; externally peer reviewed.

Data sharing statement No additional data available.

\section{REFERENCES}

1. Matsuda R. Life-style choices and falls. In: Kondo K, ed, Health Inequalities in Japan: An Empirical Study of Older People. Melbourne, Australia: Trans Pacific Press, 2010.

2. Tinetti ME, Speechley M, Ginter SF. Risk factors for falls among elderly persons living in the community. $N$ Engl J Med 1988;319:1701-7.

3. Nevitt MC, Cummings SR, Kidd S, et al. Risk factors for recurrent nonsyncopal falls: a prospective study. JAMA 1989;261:2663-8.

4. Department of Economic and Social Affairs, United Nations. World Population Ageing: 1950-2050. http://www.un.org/esa/population/ publications/worldageing19502050/

5. Hayashi Y. [Prevention of the falls in the elderly] (In Japanese). Nihon Ronen Igakkai Zasshi 2007;44:591-4.

6. Anon. Guideline for the prevention of falls in older persons. American Geriatrics Society, British Geriatrics Society, and American Academy of Orthopaedic Surgeons Panel on Falls Prevention. J Am Geriatr Soc 2001;49:664-72.

7. Ueno M, Kawai S, Mino T, et al. [Systematic review of fall-related factors among the house-dwelling elderly in Japan] (In Japanese). Nihon Ronen Igakkai Zasshi 2006;43:92-101.

8. Sherrington C, Whitney JC, Lord SR, et al. Effective exercise for the prevention of falls: a systematic review and meta-analysis. J Am Geriatr Soc 2008;56:2234-43.

9. Shaw FE, Bond J, Richardson DA, et al. Multifactorial intervention after a fall in older people with cognitive impairment and dementia presenting to the accident and emergency department: randomised controlled trial. BMJ 2003:326:73-5.

10. Gates S, Fisher JD, Cooke MW, et al. Multifactorial assessment and targeted intervention for preventing falls and injuries among older people in community and emergency care settings: systematic review and meta-analysis. BMJ 2008;336:130-3.
11. Bracco P, Deregibus A, Piscetta R. Effects of different jaw relations on postural stability in human subjects. Neurosci Lett 2004;356:228-30.

12. Yamaga $\mathrm{T}$, Yoshihara $\mathrm{A}$, Ando $\mathrm{Y}$, et al. Relationship between denta occlusion and physical fitness in an elderly population. J Gerontol $A$ Biol Sci Med Sci 2002;57:M616-20.

13. Okuyama N, Yamaga T, Yoshihara A, et al. Influence of dental occlusion on physical fitness decline in a healthy Japanese elderly population. Arch Gerontol Geriatr 2011;52:172-6.

14. Yoshida $M$, Morikawa $H$, Kanehisa $Y$, et al. Functional dental occlusion may prevent falls in elderly individuals with dementia. $\mathrm{J} \mathrm{Am}$ Geriatr Soc 2005;53:1631-2.

15. Gangloff P, Louis JP, Perrin PP. Dental occlusion modifies gaze and posture stabilization in human subjects. Neurosci Lett 2000;293:203-6.

16. Kondo K. Introducing a survey on health of older people in Japan: AGES (Aichi Gerontological Evaluation Study) project. In: Kondo K, ed, Health Inequalities in Japan: An Empirical Study of Older People. Melbourne, Australia: Trans Pacific Press, 2010.

17. Nishi A, Kondo K, Hirai H, et al. Cohort profile: the AGES 2003 cohort study in Aichi, Japan. J Epidemiol 2011;21:151-7.

18. Aida J, Hanibuchi T, Nakade M, et al. The different effects of vertical social capital and horizontal social capital on dental status: a multilevel analysis. Soc Sci Med 2009;69:512-18.

19. Lord SR, Clark RD, Webster IW. Physiological factors associated with falls in an elderly population. J Am Geriatr Soc 1991;39:1194-200.

20. Ivers $R Q$, Cumming $R G$, Mitchell $P$, et al. Visual impairment and falls in older adults: the Blue Mountains Eye Study. J Am Geriatr Soc 1998;46:58-64.

21. Grundstrom AC, Guse CE, Layde PM. Risk factors for falls and fallrelated injuries in adults 85 years of age and older. Arch Gerontol Geriatr 2012;54:421-8.

22. Marshall SW, Runyan CW, Yang J, et al. Prevalence of selected risk and protective factors for falls in the home. Am J Prev Med 2005;28:95-101.

23. Murata $\mathrm{C}$, Kondo $\mathrm{T}$, Tamakoshi $\mathrm{K}$, et al. Factors associated with life space among community-living rural elders in Japan. Public Health Nurs 2006;23:324-31.

24. WHO Expert Consultation. Appropriate body-mass index for Asian populations and its implications for policy and intervention strategies. Lancet 2004:363:157-63.

25. Burke WJ, Roccaforte WH, Wengel SP. The short form of the Geriatric Depression Scale: a comparison with the 30 -item form. $J$ Geriatr Psychiatry Neurol 1991;4:173-8.

26. Aida J, Kondo $\mathrm{K}$, Hirai $\mathrm{H}$, et al. Association of dental status and incident disability among an older Japanese population. J Am Geriatr Soc 2012;60:338-43.

27. Maruya M, Shimizu K, Ohnuma T, et al. [The effect of wearing denture and changes of occlusal position on body sway in edentulous patient] (In Japanese). J Jpn Prosthodont Soc 2000;44:781-5.

28. Käyser AF. Shortened dental arches and oral function. J Oral Rehabil 1981;8:457-62.

29. Yoshihara $A$, Watanabe $R$, Nishimuta $M$, et al. The relationship between dietary intake and the number of teeth in elderly Japanese subjects. Gerodontology 2005;22:211-18.

30. Takata Y, Ansai T, Awano S, et al. Relationship of physical fitness to chewing in an 80-year-old population. Oral Dis 2004;10:44-9.

31. Fukai K, Takiguchi T, Ando Y, et al. Critical tooth number without subjective dysphagia. Geriatr Gerontol Int 2011;11:482-7.

32. Miyaura K, Morita M, Matsuka $Y$, et al. Rehabilitation of biting abilities in patients with different types of dental prostheses. J Oral Rehabil 2000;27:1073-6.

33. Moreland J, Richardson J, Chan DH, et al. Evidence-based guidelines for the secondary prevention of falls in older adults. Gerontology 2003;49:93-116.

34. Pitiphat W, Garcia RI, Douglass CW, et al. Validation of self reported oral health measures. J Public Health Dent 2002;62:122-8.

35. Douglass CW, Berlin J, Tennstedt S. The validity of self-reported ora health status in the elderly. J Public Health Dent 1991;51:220-2.

36. Ando $Y$, Ikeda S, Yoshihara A. [The reliability of self-assessment of number of remaining teeth using questionnaires] (In Japanese). J Dent Health 1997;47:657-62.

37. Cummings SR, Nevitt MC, Kidd S. Forgetting falls. The limited accuracy of recall of falls in the elderly. J Am Geriatr Soc 1988;36:613-16. 